\title{
A!
}

This is an electronic reprint of the original article.

This reprint may differ from the original in pagination and typographic detail.

Awan, Hafiz Asad Ali; Saarakkala, Seppo E.; Hinkkanen, Marko

\section{Current control of saturated synchronous motors}

Published in:

Proceedings of the 10th IEEE Energy Conversion Congress and Exposition, ECCE 2018

DOI:

10.1109/ECCE.2018.8558120

Published: 23/09/2018

Document Version

Peer reviewed version

Please cite the original version:

Awan, H. A. A., Saarakkala, S. E., \& Hinkkanen, M. (2018). Current control of saturated synchronous motors. In

Proceedings of the 10th IEEE Energy Conversion Congress and Exposition, ECCE 2018 (pp. 6953-6959). (IEEE

Energy Conversion Congress and Exposition). IEEE. https://doi.org/10.1109/ECCE.2018.8558120

This material is protected by copyright and other intellectual property rights, and duplication or sale of all or part of any of the repository collections is not permitted, except that material may be duplicated by you for your research use or educational purposes in electronic or print form. You must obtain permission for any other use. Electronic or print copies may not be offered, whether for sale or otherwise to anyone who is not an authorised user. 


\title{
Current Control of Saturated Synchronous Motors
}

\author{
Hafiz Asad Ali Awan, Seppo E. Saarakkala, and Marko Hinkkanen \\ Aalto University School of Electrical Engineering \\ Espoo, Finland
}

\begin{abstract}
Synchronous reluctance motors (SyRMs) with or without permanent magnets (PMs) have highly nonlinear saturation characteristics. The effects of the magnetic saturation can be included in the current controller by changing its state variable from the stator current to the stator flux linkage using the known saturation characteristics. If the saturation characteristics are linear, the proposed control structure reduces to the standard current controller in this special case. Experimental results on a 6.7-kW SyRM drive demonstrate that the proposed controller enables a higher closed-loop bandwidth as compared to the standard current controller.

Index Terms-Current control, magnetic saturation, permanent-magnet synchronous motor, synchronous reluctance motor.
\end{abstract}

\section{INTRODUCTION}

Highly saturated synchronous motors, such as synchronous reluctance motors (SyRMs) with or without permanent magnets (PMs), have good flux-weakening capability, high torque density, and wide constant power region. The maximum operating frequencies of these motors can be very high, e.g., 12000 $\mathrm{r} / \mathrm{min}$ corresponding to the frequency of $1000 \mathrm{~Hz}$ for a tenpole machine. A high-performance current controller is needed to optimally control these drives. Typically, the stator current is controlled in measured or estimated rotor coordinates [1]-[10], as illustrated in Fig. 1. In order to reach very high fundamental frequencies at low sampling frequencies, direct discrete-time designs are preferred [5], [7], [8].

The high torque density of SyRMs and PM-SyRMs comes at a price of highly nonlinear saturation characteristics. Therefore, it is necessary to schedule the current controller gains to depend on the operating point currents. This gain scheduling is typically implemented using the chord-slope inductances, since including the effect of the incremental inductances is complicated [8]. To avoid too large overshoots in the control response, comparatively low closed-loop bandwidths have to be used.

In control systems for saturated synchronous motor drives, the nonlinear saturation characteristics are needed, e.g., in a flux observer or for solving the maximum-torque-per-ampere locus. Therefore, the measured current and the reference current can be easily mapped to the corresponding flux linkage variables using the known saturation characteristics. These flux linkages can then be used in the current controller. If the magnetics are modeled to be linear, this kind of a modified current controller becomes mathematically equivalent to the standard current controller. However, in the case of nonlinear characteristics, the effects of the incremental inductances are

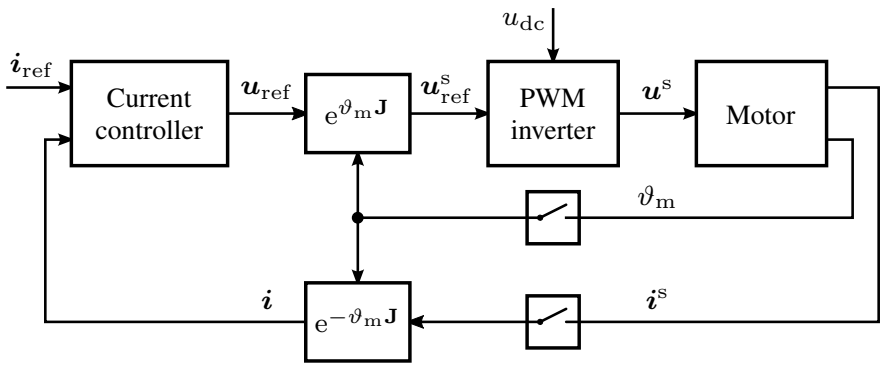

Fig. 1. Current controller operating in rotor coordinates. The quantities in stator coordinates are marked with the superscript $\mathrm{s}$.

inherently taken into account, unlike in the case of the standard current controller.

In this paper, a current control structure suitable for highly saturated synchronous motors is proposed. The main contributions are:

1) An improved current control structure is presented, based on the nonlinear change of a state variable from the current to the flux linkage. Saturation characteristics are modeled in a generic form, which makes the controller compatible with both look-up tables and explicit functions.

2) A state-feedback controller with reference feedforward and integral action is designed similarly as in [8].

3) The effects due to the sampling and hold, computational delay, and voltage saturation are also covered.

Experimental results are presented for a $6.7-\mathrm{kW}$ SyRM drive.

\section{Motor MODEL}

\section{A. Saturation Characteristics}

Generally, the stator flux linkage $\psi$ of a synchronous motor is a nonlinear function of the stator current $i$, i.e.,

$$
\boldsymbol{\psi}=\boldsymbol{\psi}(\boldsymbol{i})=\left[\begin{array}{l}
\psi_{\mathrm{d}}\left(i_{\mathrm{d}}, i_{\mathrm{q}}\right) \\
\psi_{\mathrm{q}}\left(i_{\mathrm{d}}, i_{\mathrm{q}}\right)
\end{array}\right]
$$

The reciprocity condition $\partial \psi_{\mathrm{d}} / \partial i_{\mathrm{q}}=\partial \psi_{\mathrm{q}} / \partial i_{\mathrm{d}}$ should hold, since the nonlinear inductor does not generate or dissipate energy. As an example, Fig. 2 illustrates the nonlinear saturation characteristics of a SyRM. In the special case of linear magnetics, the stator flux linkage is

$$
\boldsymbol{\psi}=\left[\begin{array}{cc}
L_{\mathrm{d}} & 0 \\
0 & L_{\mathrm{q}}
\end{array}\right] \boldsymbol{i}+\left[\begin{array}{c}
\psi_{\mathrm{f}} \\
0
\end{array}\right]
$$

with the constant inductances $L_{\mathrm{d}}$ and $L_{\mathrm{q}}$ and constant PMflux linkage $\psi_{\mathrm{f}}$. It is worth noticing that the effect of PMs 


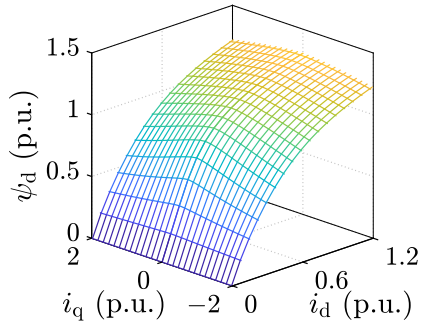

(a)

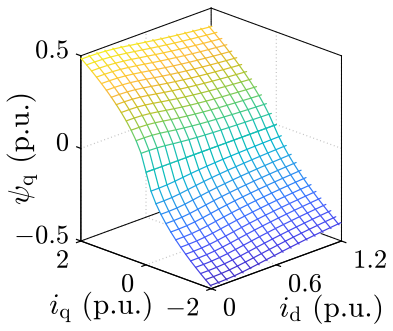

(b)

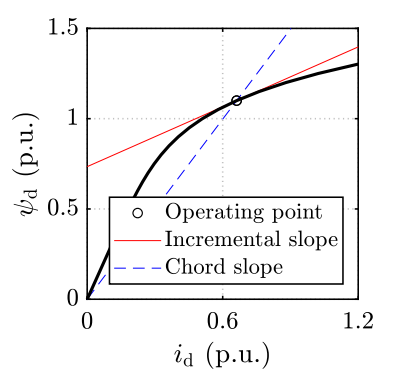

(c)

Fig. 2. Saturation characteristics of a 6.7-kW SyRM: (a) $\psi_{\mathrm{d}}=\psi_{\mathrm{d}}\left(i_{\mathrm{d}}, i_{\mathrm{q}}\right)$; (b) $\psi_{\mathrm{q}}=\psi_{\mathrm{q}}\left(i_{\mathrm{d}}, i_{\mathrm{q}}\right)$; (c) $\psi_{\mathrm{d}}=\psi_{\mathrm{d}}\left(i_{\mathrm{d}}, 0\right)$.

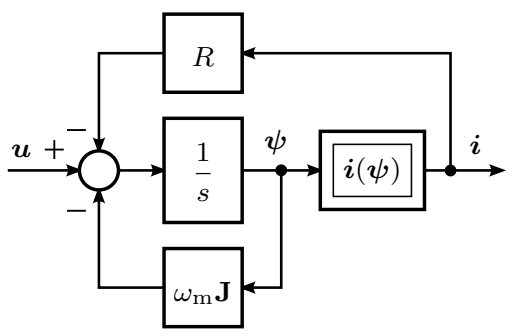

Fig. 3. Model of a saturated synchronous motor in rotor coordinates.

is inherently included in (1). Alternatively, the saturation characteristics can be defined using the inverse function of (1), i.e.,

$$
\boldsymbol{i}=\boldsymbol{i}(\boldsymbol{\psi})=\left[\begin{array}{l}
i_{\mathrm{d}}\left(\psi_{\mathrm{d}}, \psi_{\mathrm{q}}\right) \\
i_{\mathrm{q}}\left(\psi_{\mathrm{d}}, \psi_{\mathrm{q}}\right)
\end{array}\right]
$$

In this paper, the saturation is modeled using generic saturation characteristics in the form of (1) or (3). The advantage of these forms is that they are directly compatible with both lookup tables and explicit functions. Furthermore, the definition of the chord-slop inductance, illustrated in Fig. 2(c), becomes ambiguous, if the machine is equipped with PMs.

\section{B. Voltage Equation}

The voltage equation of a synchronous motor in rotor coordinates is

$$
\frac{\mathrm{d} \boldsymbol{\psi}}{\mathrm{d} t}=\boldsymbol{u}-R \boldsymbol{i}-\omega_{\mathrm{m}} \mathbf{J} \boldsymbol{\psi}
$$

where $\boldsymbol{u}$ is the stator voltage, $R$ is the stator resistance, $\omega_{\mathrm{m}}$ is the electrical angular speed of the rotor, and $\mathbf{J}=\left[\begin{array}{cc}0 & -1 \\ 1 & 0\end{array}\right]$ is the orthogonal rotation matrix. The voltage equation (4) together

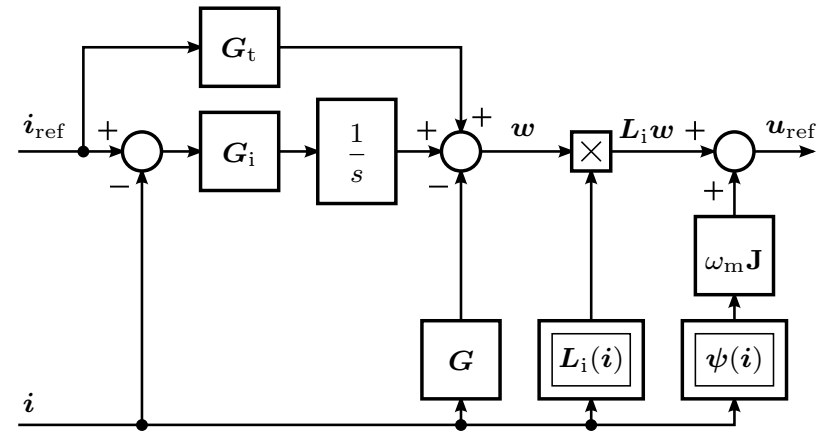

(a)

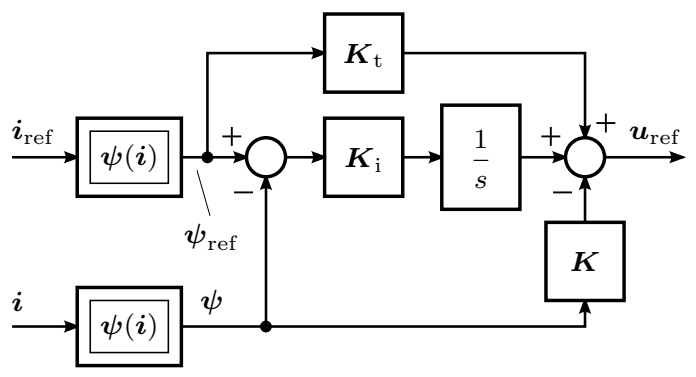

(b)

Fig. 4. Current controllers: (a) current as a state variable; (b) flux linkage as a state variable.

with the saturation characteristics (3) forms a nonlinear statespace model of the motor, cf. Fig. 3.

Substituting (1) into (4) gives the voltage equation with the stator current as a state variable

$$
\boldsymbol{L}_{\mathrm{i}} \frac{\mathrm{d} \boldsymbol{i}}{\mathrm{d} t}=\boldsymbol{u}-R \boldsymbol{i}-\omega_{\mathrm{m}} \mathbf{J} \psi
$$

where the incremental inductance matrix is

$$
\begin{aligned}
\boldsymbol{L}_{\mathrm{i}}=\boldsymbol{L}_{\mathrm{i}}(\boldsymbol{i}) & =\left[\begin{array}{ll}
\frac{\partial \psi_{\mathrm{d}}\left(i_{\mathrm{d}}, i_{\mathrm{q}}\right)}{\partial i_{\mathrm{d}}} & \frac{\partial \psi_{\mathrm{d}}\left(i_{\mathrm{d}}, i_{\mathrm{q}}\right)}{\partial i_{\mathrm{q}}} \\
\frac{\partial \psi_{\mathrm{q}}\left(i_{\mathrm{d}}, i_{\mathrm{q}}\right)}{\partial i_{\mathrm{d}}} & \frac{\partial \psi_{\mathrm{q}}\left(i_{\mathrm{d}}, i_{\mathrm{q}}\right)}{\partial i_{\mathrm{q}}}
\end{array}\right] \\
& =\left[\begin{array}{ll}
L_{\mathrm{di}}\left(i_{\mathrm{d}}, i_{\mathrm{q}}\right) & L_{\mathrm{dqi}}\left(i_{\mathrm{d}}, i_{\mathrm{q}}\right) \\
L_{\mathrm{dqi}}\left(i_{\mathrm{d}}, i_{\mathrm{q}}\right) & L_{\mathrm{qi}}\left(i_{\mathrm{d}}, i_{\mathrm{q}}\right)
\end{array}\right]
\end{aligned}
$$

The matrix is symmetric due to the reciprocity condition. Fig. 2(c) illustrates the definition of the incremental inductance. If the generic saturation characteristics are known, the definition of the incremental inductance is unambiguous in all operating point (unlike the definition of the chord-slope inductance).

\section{Continuous-Time Current Control Design}

Fig. 1 shows the current controller, which operates in rotor coordinates. The electrical angular position of the rotor is denoted by $\vartheta_{\mathrm{m}}$. In this section, the pulse-width modulator (PWM) is considered to be ideal, $\boldsymbol{u}=\boldsymbol{u}_{\text {ref }}$. The effects of the voltage saturation and of the time delays are considered later in Section IV. For simplicity, the current controllers are tuned assuming the stator resistance $R=0$, i.e., the effect of the resistive voltage drop is compensated for by the integral control action. 


\section{A. Current as a State Variable}

Fig. 4(a) shows the current control structure, where the magnetic saturation is taken into account based on (5). The voltage reference is given by

$$
\boldsymbol{u}_{\mathrm{ref}}=\omega_{\mathrm{m}} \mathbf{J} \psi+\boldsymbol{L}_{\mathrm{i}} \boldsymbol{w}
$$

where $\boldsymbol{w}$ is the output from a linear controller. Assuming $\boldsymbol{u}=$ $\boldsymbol{u}_{\text {ref }}$ and $R=0$ leads to

$$
\frac{\mathrm{d} \boldsymbol{i}}{\mathrm{d} t}=\boldsymbol{w}
$$

The closed-loop poles can now be easily placed via $\boldsymbol{w}$. Here, a state-feedback controller with integral action and reference feedforward is used,

$$
\boldsymbol{w}=\boldsymbol{G}_{\mathrm{t}} \boldsymbol{i}_{\mathrm{ref}}+\frac{\boldsymbol{G}_{\mathrm{i}}}{s}\left(\boldsymbol{i}_{\mathrm{ref}}-\boldsymbol{i}\right)-\boldsymbol{G} \boldsymbol{i}
$$

where $s=\mathrm{d} / \mathrm{d} t$ is the differential operator, $\boldsymbol{G}_{\mathrm{t}}$ is the reference-feedforward gain, $G_{\mathrm{i}}$ is the integral gain, and $G$ is the state-feedback gain. These gains can be chosen in various ways. As an example, choosing $\boldsymbol{G}_{\mathrm{t}}=\alpha \mathbf{I}, \boldsymbol{G}_{\mathrm{i}}=\alpha^{2} \mathbf{I}$, and $\boldsymbol{G}=2 \alpha \mathbf{I}$, where $\mathbf{I}=\left[\begin{array}{ll}1 & 0 \\ 0 & 1\end{array}\right]$ is the identity matrix, corresponds to the internal model control (IMC) design [1]. In this case, all the closed-loop poles are placed at $s=-\alpha$. The closed-loop reference-tracking dynamics are

$$
\boldsymbol{i}=\frac{\alpha}{s+\alpha} \boldsymbol{i}_{\mathrm{ref}}
$$

where $\alpha$ is the bandwidth.

For implementing the control law (7), five nonlinear functions $\psi_{\mathrm{d}}\left(i_{\mathrm{d}}, i_{\mathrm{q}}\right), \psi_{\mathrm{q}}\left(i_{\mathrm{d}}, i_{\mathrm{q}}\right), L_{\mathrm{di}}\left(i_{\mathrm{d}}, i_{\mathrm{q}}\right), L_{\mathrm{qi}}\left(i_{\mathrm{d}}, i_{\mathrm{q}}\right)$, and $L_{\mathrm{dqi}}\left(i_{\mathrm{d}}, i_{\mathrm{q}}\right)$ should be implemented, typically with look-up tables, which complicates the control system. Furthermore, delays of the discrete-time implementation cause errors in the modeled incremental inductances, especially if the sampling frequency is low.

\section{B. Flux Linkage as a State Variable}

Fig. 4(b) shows the current control structure, where the flux linkage is chosen as a state variable. Similarly to (9), a state-feedback controller with integral action and reference feedforward is used,

$$
\boldsymbol{u}_{\text {ref }}=\boldsymbol{K}_{\mathrm{t}} \boldsymbol{\psi}_{\text {ref }}+\frac{\boldsymbol{K}_{\mathrm{i}}}{s}\left(\boldsymbol{\psi}_{\text {ref }}-\boldsymbol{\psi}\right)-\boldsymbol{K} \boldsymbol{\psi}
$$

where $\boldsymbol{K}_{\mathrm{t}}$ is the reference-feedforward gain, $\boldsymbol{K}_{\mathrm{i}}$ is the integral gain, and $\boldsymbol{K}$ is the state-feedback gain. ${ }^{1}$ Two typical selections for the these gains are the IMC design [1]

$$
\boldsymbol{K}_{\mathrm{t}}=\alpha \mathbf{I} \quad \boldsymbol{K}_{\mathrm{i}}=\alpha^{2} \mathbf{I} \quad \boldsymbol{K}=2 \alpha \mathbf{I}-\omega_{\mathrm{m}} \mathbf{J}
$$

and the complex vector design [3]

$$
\boldsymbol{K}_{\mathrm{t}}=\alpha \mathbf{I} \quad \boldsymbol{K}_{\mathrm{i}}=\alpha \mathbf{I}\left(\alpha \mathbf{I}+\omega_{\mathrm{m}} \mathbf{J}\right) \quad \boldsymbol{K}=2 \alpha \mathbf{I}
$$

\footnotetext{
${ }^{1}$ The controller (11) does not see the magnetic saliency of the motor due to the mapping of the current to the flux linkage. Therefore, the controller (11) and the resulting flux linkage dynamics could be described using complex space vectors and complex gains, instead of real space vectors and gain matrices.
}

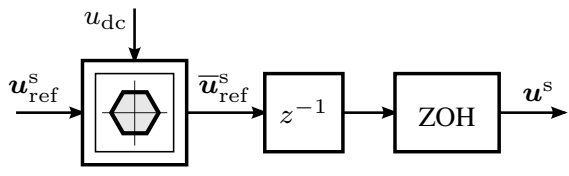

Fig. 5. Model of a PWM inverter in stator coordinates. The model consists of the voltage saturation, the computational delay $z^{-1}$, and the $\mathrm{ZOH}$.

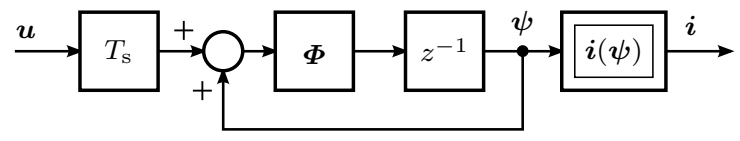

Fig. 6. Hold-equivalent motor model in rotor coordinates. The state-transition matrix is $\boldsymbol{\Phi}=\exp \left(-T_{\mathrm{s}} \omega_{\mathrm{m}} \mathbf{J}\right)$.

Both these designs lead to the first-order closed-loop system

$$
\psi=\frac{\alpha}{s+\alpha} \psi_{\text {ref }}
$$

However, the disturbance rejection characteristics of the two designs are different, leading also to different sensitivities to parameter errors [8].

In the steady state, $\psi=\psi_{\text {ref }}$ holds due to the integral action. The same saturation characteristics $\boldsymbol{\psi}=\boldsymbol{\psi}(\boldsymbol{i})$ is used to map both the reference current and the actual current to the corresponding flux linkages. Therefore, $\boldsymbol{i}=\boldsymbol{i}_{\text {ref }}$ holds in the steady state, even with inaccurate saturation characteristics. The control structure in Fig. 4(b) needs only two look-up tables, $\psi_{\mathrm{d}}\left(i_{\mathrm{d}}, i_{\mathrm{q}}\right)$ and $\psi_{\mathrm{q}}\left(i_{\mathrm{d}}, i_{\mathrm{q}}\right)$, and it inherently takes the effects of the incremental inductances into account. It is also worth noticing that the two controllers in Fig. 4 are mathematically equivalent, if the magnetic saturation is omitted and if the same pole locations are chosen.

\section{Direct Discrete-Time Control Design}

For designing the current controller directly in the discretetime domain, a hold-equivalent discrete-time model of the motor is needed. Furthermore, the limited inverter voltage has to be properly taken into account in the current controller, independently of the selected design approach.

\section{A. Hold-Equivalent Motor Model}

Fig. 5 shows the switching-cycle-averaged model of a PWM inverter in stator coordinates. The model consists of the voltage saturation (corresponding to the voltage hexagon), the computational delay of one sampling period, and the zeroorder hold $(\mathrm{ZOH})$. The voltage $\boldsymbol{u}^{\mathrm{s}}(t)$ in stator coordinates is kept constant between consecutive sampling instants. The effects of the voltage saturation are omitted here, but they are considered in the latter subsection. The stator resistance $R=0$ is assumed. Under these assumptions, the exact holdequivalent discrete-time model in rotor coordinates can be derived from (4), leading to

$$
\boldsymbol{\psi}(k+1)=\boldsymbol{\Phi} \boldsymbol{\psi}(k)+T_{\mathrm{s}} \boldsymbol{\Phi} \boldsymbol{u}(k)
$$

where $T_{\mathrm{s}}$ is the sampling period. The state-transition matrix is

$$
\boldsymbol{\Phi}=\exp \left(-T_{\mathrm{s}} \omega_{\mathrm{m}} \mathbf{J}\right)
$$




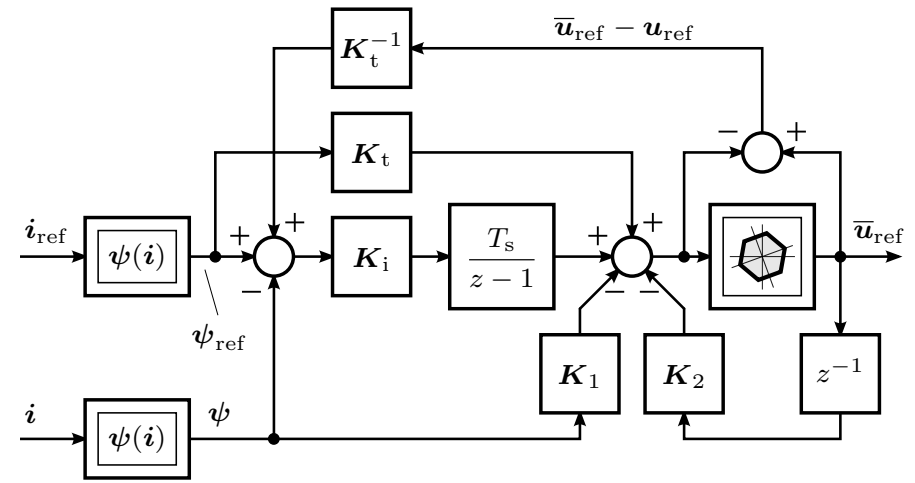

Fig. 7. Discrete-time current controller. The voltage saturation and the antiwindup mechanism are also shown. The limited voltage reference $\overline{\boldsymbol{u}}_{\text {ref }}$ can be calculated as a part of the current control algorithm or it can be provided by the PWM algorithm. In the linear modulation range, $\overline{\boldsymbol{u}}_{\mathrm{ref}}=\boldsymbol{u}_{\mathrm{ref}}$.

As can be seen in Fig. 6, the nonlinear saturation characteristics appear only in the output equation due to the assumption $R=0$. Therefore, generic nonlinear saturation characteristics $\boldsymbol{i}=\boldsymbol{i}(\boldsymbol{\psi})$ can be used. The assumption $R=0$ also simplifies the discrete-time model.

Due to the finite computational time, the digital control system and the PWM update typically have one samplingperiod time delay, i.e, $\boldsymbol{u}^{\mathrm{s}}(k)=\boldsymbol{u}_{\text {ref }}^{\mathrm{s}}(k-1)$. For control design, the computational delay can be easily included in the plant model as follows

$$
\left[\begin{array}{c}
\boldsymbol{\psi}(k+1) \\
\boldsymbol{u}(k+1)
\end{array}\right]=\left[\begin{array}{cc}
\boldsymbol{\Phi} & T_{\mathrm{S}} \boldsymbol{\Phi} \\
\mathbf{O} & \mathbf{O}
\end{array}\right]\left[\begin{array}{l}
\boldsymbol{\psi}(k) \\
\boldsymbol{u}(k)
\end{array}\right]+\left[\begin{array}{l}
\mathbf{O} \\
\boldsymbol{\Phi}
\end{array}\right] \boldsymbol{u}_{\mathrm{ref}}(k)
$$

where $\mathbf{O}=\left[\begin{array}{ll}0 & 0 \\ 0 & 0\end{array}\right]$ is the zero matrix. Due to this computational delay, the order of the discrete-time plant model is higher than the continuous-time model. An advantage of the discrete-time control design is that the delay can be easily taken into account in the controller, as discussed in the following.

\section{B. Control Design}

Similarly to the continuous-time design, the stator currents are mapped to the flux linkages. Furthermore, a state-feedback controller with integral action and reference feedforward is applied [8]. The control algorithm is

$$
\begin{aligned}
& \boldsymbol{u}_{\mathrm{i}}(k+1)= \boldsymbol{u}_{\mathrm{i}}(k)+T_{\mathrm{s}} \boldsymbol{K}_{\mathrm{i}}\left[\boldsymbol{\psi}_{\mathrm{ref}}(k)-\boldsymbol{\psi}(k)\right] \\
& \boldsymbol{u}_{\mathrm{ref}}(k)=\boldsymbol{K}_{\mathrm{t}} \boldsymbol{\psi}_{\mathrm{ref}}(k)-\boldsymbol{K}_{1} \boldsymbol{\psi}(k) \\
& \quad-\boldsymbol{K}_{2} \boldsymbol{u}_{\mathrm{ref}}(k-1)+\boldsymbol{u}_{\mathrm{i}}(k)
\end{aligned}
$$

where $\boldsymbol{u}_{\mathrm{i}}$ is the integral state, $\boldsymbol{K}_{\mathrm{i}}$ is the integral gain, $\boldsymbol{K}_{\mathrm{t}}$ is the feedforward gain, and $\boldsymbol{K}_{1}$ and $\boldsymbol{K}_{2}$ are the state-feedback gains. Fig. 7 shows the corresponding block diagram, where also the anti-windup mechanism is included. It can be seen that the discrete-time control design is very similar to the continuous-time design. The computational delay is omitted in the continuous-time design, while it is taken into account in the discrete-time design by means of $\boldsymbol{K}_{2}$.

Based on (17) and (18), the closed-loop reference-tracking dynamics can be expressed as

$$
\boldsymbol{\psi}(k)=\left(z^{3} \mathbf{I}+z^{2} \boldsymbol{A}_{2}+z \boldsymbol{A}_{1}+\boldsymbol{A}_{0}\right)^{-1}\left(z \boldsymbol{B}_{1}+\boldsymbol{B}_{0}\right) \boldsymbol{\psi}_{\text {ref }}(k)
$$

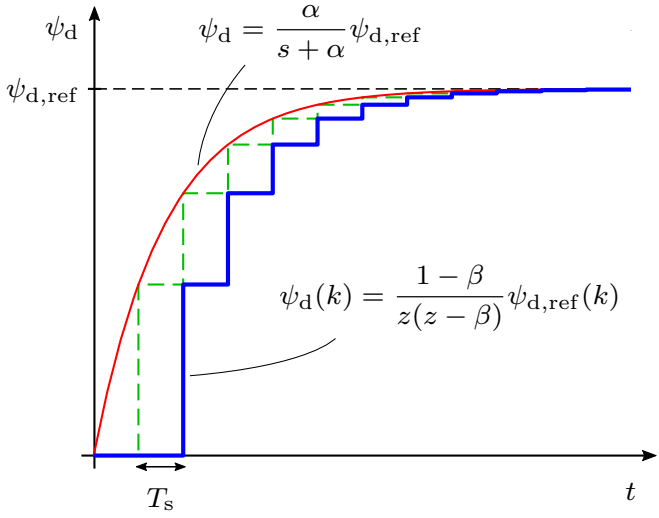

Fig. 8. Step responses of the continuous-time system (14) and the discretetime system (23), where $\beta=\exp \left(-\alpha T_{\mathrm{S}}\right)$. Setting $\beta=0$ would give the dead-beat response. The green dashed line shows the response of the discretetime system without the computational delay.

where $\boldsymbol{A}_{0}, \boldsymbol{A}_{1}, \boldsymbol{A}_{2}, \boldsymbol{B}_{0}$, and $\boldsymbol{B}_{1}$ are the coefficient matrices. The gain matrices can be solved as functions of the coefficient matrices

$$
\begin{gathered}
\boldsymbol{K}_{\mathrm{t}}=\frac{\boldsymbol{\Phi}^{-2} \boldsymbol{B}_{1}}{T_{\mathrm{s}}} \quad \boldsymbol{K}_{\mathrm{i}}=\frac{\boldsymbol{\Phi}^{-2}\left(\mathbf{I}+\boldsymbol{A}_{0}+\boldsymbol{A}_{1}+\boldsymbol{A}_{2}\right)}{T_{\mathrm{s}}^{2}} \\
\boldsymbol{K}_{1}=\frac{\mathbf{I}+\boldsymbol{\Phi}^{-2}\left(\mathbf{I}+\boldsymbol{\Phi}+\boldsymbol{A}_{1}+\boldsymbol{A}_{2}+\boldsymbol{A}_{2} \boldsymbol{\Phi}\right)}{T_{\mathrm{s}}} \\
\boldsymbol{K}_{2}=\mathbf{I}+\boldsymbol{\Phi}+\boldsymbol{\Phi}^{-2} \boldsymbol{A}_{2} \boldsymbol{\Phi}^{2}
\end{gathered}
$$

Using these expressions, the poles and transmission zeros of (19) can be arbitrarily placed. Due to the time delay, $\boldsymbol{A}_{0}=$ $\mathbf{O}$ is preferably selected. The discrete-time counterpart of the IMC design is defined by

$$
\boldsymbol{A}_{1}=\beta^{2} \mathbf{I} \quad \boldsymbol{A}_{2}=-2 \beta \mathbf{I} \quad \boldsymbol{B}_{1}=(1-\beta) \mathbf{I}
$$

and the complex vector design by

$$
\boldsymbol{A}_{1}=\beta^{2} \boldsymbol{\Phi} \quad \boldsymbol{A}_{2}=-\beta(\mathbf{I}+\boldsymbol{\Phi}) \quad \boldsymbol{B}_{1}=(1-\beta) \mathbf{I}
$$

where $\beta=\exp \left(-\alpha T_{\mathrm{s}}\right)$ is the exact mapping in the discrete domain of the intended real pole of the system. In both cases, the closed-loop transfer-function matrix (19) reduces to

$$
\boldsymbol{\psi}(k)=\frac{1-\beta}{z(z-\beta)} \boldsymbol{\psi}_{\mathrm{ref}}(k)
$$

The step response corresponding to (23) is shown in Fig. 8.

\section{Voltage Saturation and Anti-Windup}

Fig. 9 illustrates the maximum available voltage, which corresponds to the border of the voltage hexagon. In the first sector, the maximum voltage magnitude is [11]

$$
u_{\max }=\frac{u_{\mathrm{dc}}}{\sqrt{3} \sin \left(2 \pi / 3-\vartheta_{\mathrm{u}}\right)}
$$

where $\vartheta_{\mathrm{u}}=[0, \pi / 3]$ is the angle of the reference voltage $\boldsymbol{u}_{\text {ref }}^{\mathrm{s}}$. This equation can be easily applied in other sectors as well. The realizable voltage reference can be calculated as

$$
\overline{\boldsymbol{u}}_{\mathrm{ref}}= \begin{cases}\boldsymbol{u}_{\mathrm{ref}}, & \text { if }\left\|\boldsymbol{u}_{\mathrm{ref}}\right\| \leq u_{\max } \\ \frac{\boldsymbol{u}_{\mathrm{ref}}}{\left\|\boldsymbol{u}_{\mathrm{ref}}\right\|} u_{\max }, & \text { if }\left\|\boldsymbol{u}_{\mathrm{ref}}\right\|>u_{\max }\end{cases}
$$




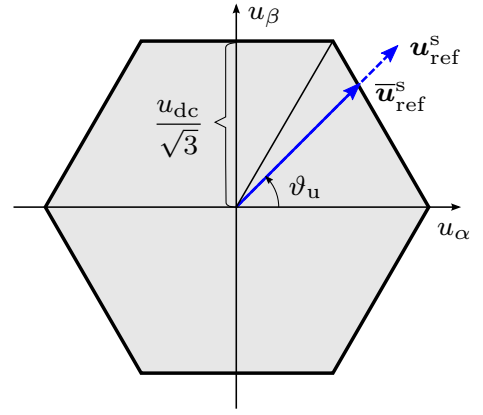

Fig. 9. Voltage hexagon of a two-level PWM inverter in stator coordinates.

Fig. 7 shows an integrator anti-windup mechanism, which is based on the realizable voltage reference $\overline{\boldsymbol{u}}_{\text {ref }}$ [12]. This mechanism is disabled in the linear modulation range where $\overline{\boldsymbol{u}}_{\text {ref }}=\boldsymbol{u}_{\text {ref }}$ holds. The realizable voltage reference can be either calculated in the current controller using (24) and (25) or it can be obtained from the PWM. It is also worth mentioning that the switching-cycle-averaged voltage at the $k$ th time instant is $\boldsymbol{u}(k)=\boldsymbol{\Phi} \overline{\boldsymbol{u}}_{\text {ref }}(k-1)$, according to (17). This signal is typically needed in the flux observer or in other estimation algorithms. Naturally, the proposed controller could also be used to control the estimated current or the estimated flux linkage (instead of the measured current).

\section{REsults}

The current controllers corresponding to Figs. 4 and 7 were evaluated by experiments. The controllers were implemented on a dSPACE DS1006 processor board. The studied motor is a transverse-laminated $6.7-\mathrm{kW}$ four-pole SyRM, whose rated data are given in Table I. The stator currents and the DC-link voltage are sampled in synchronism with the single-update PWM. The sampling (switching) frequency is $5 \mathrm{kHz}$. The desired closed-loop bandwidth of the controllers is $2 \pi \cdot 200$ $\mathrm{rad} / \mathrm{s}$, unless stated otherwise. A servo induction machine is used to regulate the speed in the constant-speed tests. Three different controllers are compared:

Design 1: Continuous-time design of Fig. 4, discretized using the forward Euler approximation with the rated inductances;

Design 2: Discrete-time design of Fig. 7 with the rated inductances;

Design 3: Discrete-time design of Fig. 7 with nonlinear saturation characteristics shown in Figs. 2(a) and $2(\mathrm{~b})$.

The complex vector design is more robust against parameter errors than the IMC design [8]. Therefore, the results are only shown for the complex vector design.

\section{A. Constant-Speed Tests}

Figs. 10 and 11 show the experimental results when the current references are changed stepwise. The speed is kept at $\omega_{\mathrm{m}}=0.5$ p.u. in Fig. 10 and at $\omega_{\mathrm{m}}=1.5$ p.u. in Fig. 11.

Figs. 10(a) and 11(a) show the results for Design 1. Significant overshoot and cross-coupling can be seen in the currents
TABLE I

DATA OF THE 6.7-KW SYRM

\begin{tabular}{lcc}
\hline Rated values & & \\
Phase voltage (peak value) & $\sqrt{2 / 3} \cdot 370 \mathrm{~V}$ & 1.00 p.u. \\
Current (peak value) & $\sqrt{2} \cdot 15.5 \mathrm{~A}$ & 1.00 p.u. \\
Frequency & $105.8 \mathrm{~Hz}$ & 1.00 p.u. \\
Speed & $3175 \mathrm{r} / \mathrm{min}$ & 1.00 p.u. \\
Torque & $20.1 \mathrm{Nm}$ & 0.67 p.u. \\
\hline Rated motor parameters & & \\
d-axis inductance $L_{\mathrm{d}}$ & $45.6 \mathrm{mH}$ & 2.20 p.u. \\
q-axis inductance $L_{\mathrm{q}}$ & $6.84 \mathrm{mH}$ & 0.33 p.u. \\
Stator resistance $R$ & $0.55 \Omega$ & 0.04 p.u. \\
PM flux $\psi_{\mathrm{f}}$ & 0 & 0 \\
\hline
\end{tabular}

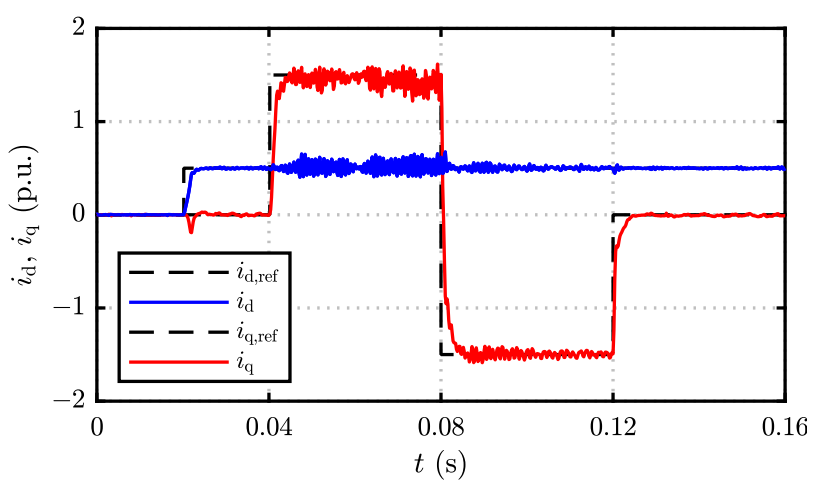

(a)

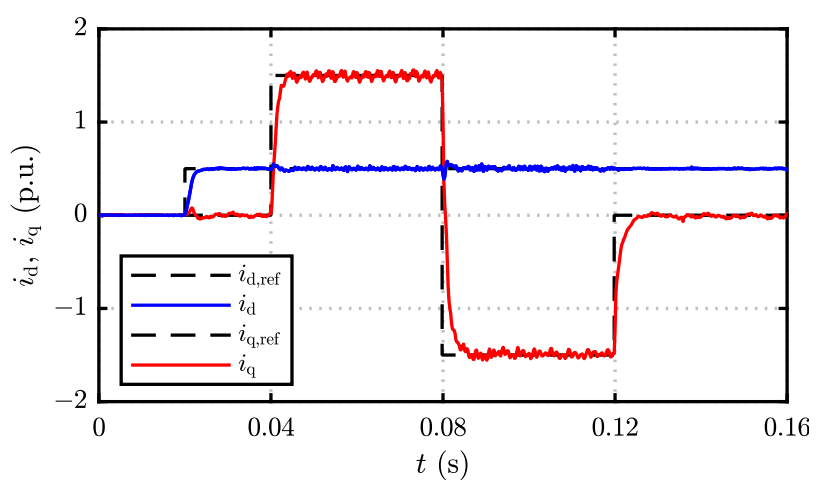

(b)

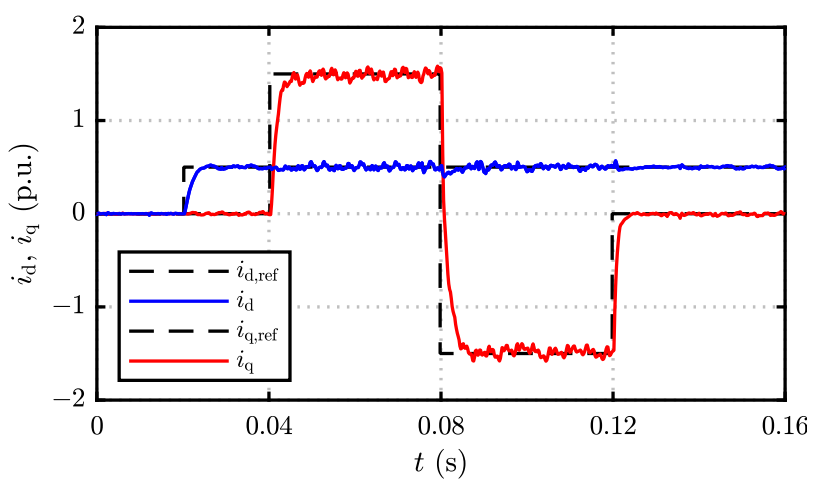

(c)

Fig. 10. Experimental results at $\omega_{\mathrm{m}}=0.5$ p.u.: (a) Design 1; (b) Design 2; (c) Design 3. The measured current components $i_{\mathrm{d}}$ and $i_{\mathrm{q}}$ and their references are shown. The desired bandwidth for all the designs is $\alpha=2 \pi \cdot 200 \mathrm{rad} / \mathrm{s}$. 


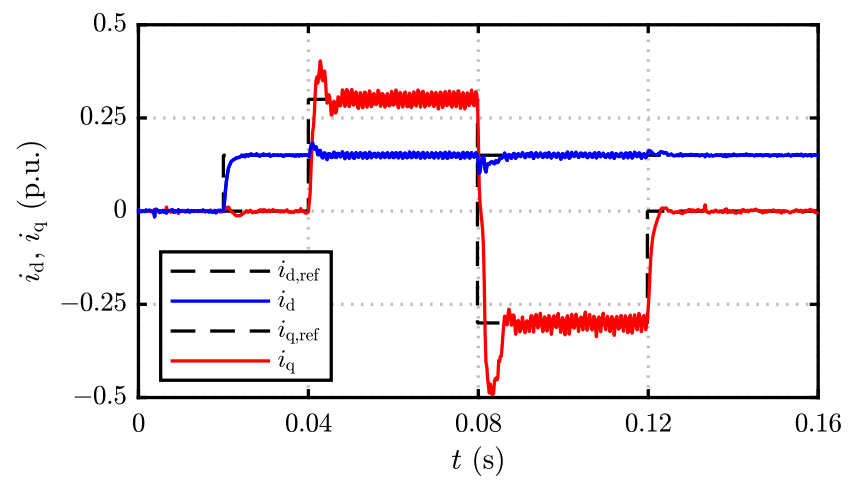

(a)

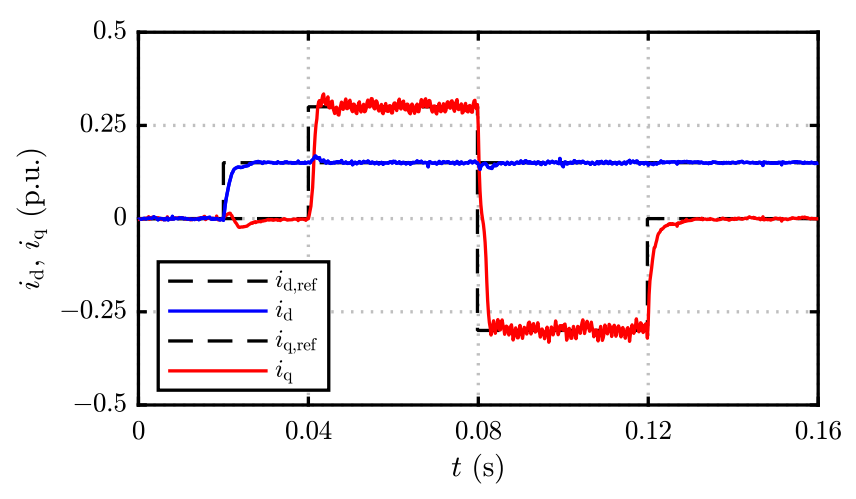

(b)

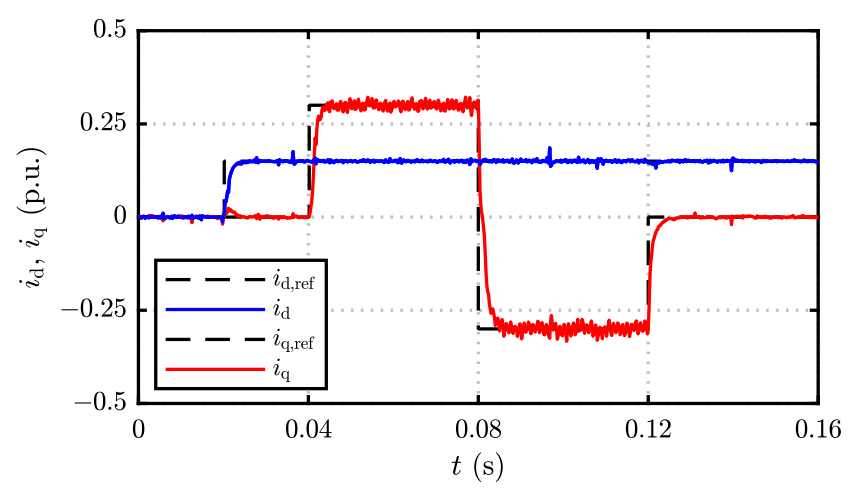

(c)

Fig. 11. Experimental results at $\omega_{\mathrm{m}}=1.5$ p.u.: (a) Design 1; (b) Design 2; (c) Design 3. The desired bandwidth of the controller for all designs is $\alpha=2 \pi \cdot 200 \mathrm{rad} / \mathrm{s}$.

after the step changes in the current references. This is an expected result since the magnetic saturation and the discretetime effects are not properly taken into account in Design 1.

Figs. 10(b) and 11(b) show the results for Design 2. This design provides better results than Design 1, as the discretetime effects are properly modeled. Still some cross-coupling and ripple in the current components can be seen since the effect of the magnetic saturation is omitted.

Figs. 10(c) and 11(c) show the results for Design 3. It can be seen in that Design 3 provides slightly better performance

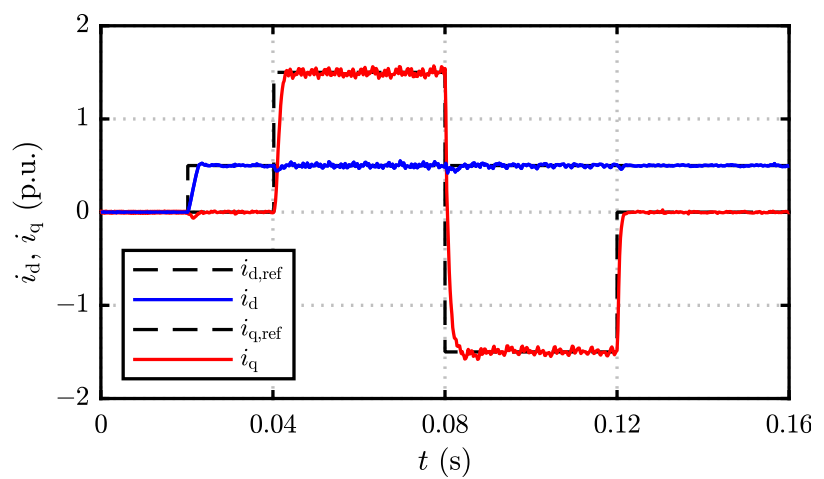

(a)

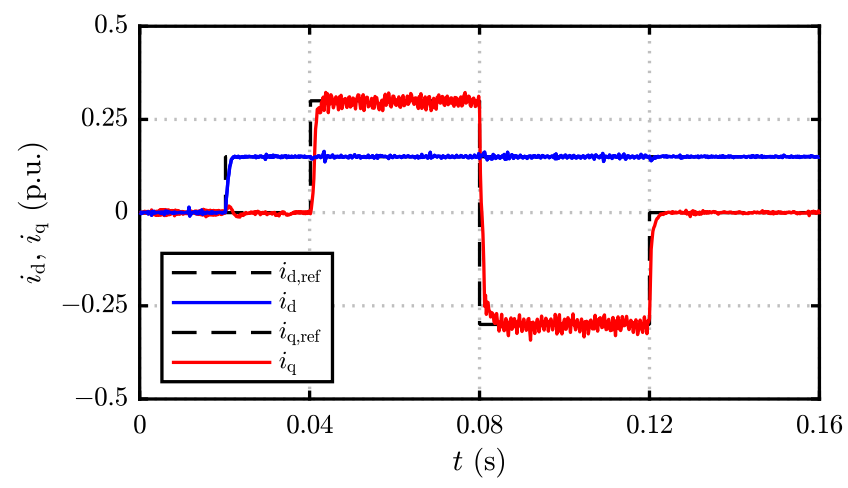

(b)

Fig. 12. Experimental results for the Design 3 at: (a) $\omega_{\mathrm{m}}=0.5$ p.u.; (b) $\omega_{\mathrm{m}}=1.5 \mathrm{p}$.u. The desired bandwidth of the controller is $\alpha=2 \pi \cdot 500 \mathrm{rad} / \mathrm{s}$.

than Design 2. If the sampling frequency were lowered or the bandwidth increased, the difference between Schemes 2 and 3 becomes even more clear.

All three designs were also evaluated at the bandwidth of $\alpha=2 \pi \cdot 500 \mathrm{rad} / \mathrm{s}$. Design 1 is unstable at this high bandwidth. The response of Design 2 has significant overshoots and crosscouplings as well as increase in the ripple content. Fig. 12(a) shows the experimental results for Design 3 at $\omega_{\mathrm{m}}=0.5$ p.u. and Fig. 12(b) at $\omega_{\mathrm{m}}=1.5$ p.u. It can be seen in Fig. 12 that almost no cross-coupling and overshoot exist.

\section{B. Acceleration Tests}

The motor is accelerated from 0 to the speed of 2 p.u. with the maximum available torque, when the current limit is 1.5 p.u. The optimal current references are calculated using the method available in [13]. Design 1 is unstable at this bandwidth of $\alpha=2 \pi \cdot 200 \mathrm{rad} / \mathrm{s}$ and significant ringing in current components is observed for Design 2. Experimental result for high speed acceleration are only shown for Design 3 , cf. Fig. 13. The speed reference is stepped from 0 to 2 p.u. at $t=0.5 \mathrm{~s}$. It can be seen that the measured current components follow their references very well. It is also worth mentioning that much higher bandwidths could be used for Design 3. 

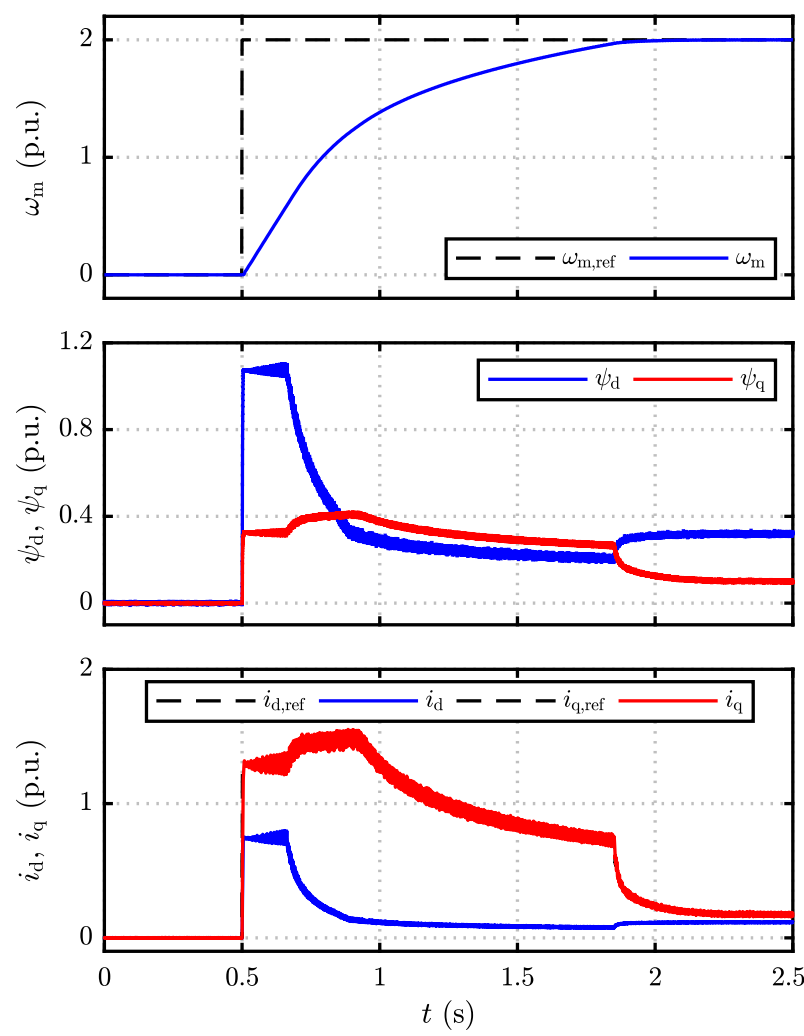

Fig. 13. Experimental results showing acceleration from zero to 2-p.u. speed for the Design 3. The first subplot shows the reference speed $\omega_{\mathrm{m} \text {,ref }}$ and the measured speed $\omega_{\mathrm{m}}$. The second subplot shows the flux components $\psi_{\mathrm{d}}$ and $\psi_{\mathrm{q}}$. The last subplot shows the measured current components $i_{\mathrm{d}}$ and $i_{\mathrm{q}}$.

\section{CONCLUSIONS}

In this paper, we presented different current control structures for taking the magnetic saturation effects into account. A simple structure is achieved via a nonlinear change of a controller state variable, i.e., the stator current is mapped to the flux linkage using the known saturation characteristics. A discrete-time equivalent of the proposed structure is also presented. If the discrete-time and saturation effects are properly taken into account, higher closed-loop bandwidths and lower sampling frequencies can be achieved. The presented controllers have been experimentally evaluated using a 6.7kW SyRM drive.

\section{REFERENCES}

[1] L. Harnefors and H.-P. Nee, "Model-based current control of AC machines using the internal model control method," IEEE Trans. Ind. Appl., vol. 34, no. 1, pp. 133-141, Jan./Feb. 1998.

[2] J. O. Krah and J. Holtz, "High-performance current regulation and efficient PWM implementation for low-inductance servo motors," IEEE Trans. Ind. Appl., vol. 35, no. 5, pp. 1039-1049, Sept./Oct. 1999.

[3] F. Briz del Blanco, M. W. Degner, and R. D. Lorenz, "Dynamic analysis of current regulators for AC motors using complex vectors," IEEE Trans. Ind. Appl., vol. 35, no. 6, pp. 1424-1432, Nov./Dec. 1999.

[4] B.-H. Bae and S.-K. Sul, "A compensation method for time delay of full-digital synchronous frame current regulator of PWM AC drives," IEEE Trans. Ind. Appl., vol. 39, no. 3, pp. 802-810, May/June 2003.

[5] H. Kim, M. W. Degner, J. M. Guerrero, F. Briz, and R. D. Lorenz, "Discrete-time current regulator design for AC machine drives," IEEE Trans. Ind. Appl., vol. 46, no. 4, pp. 1425-1435, July/Aug. 2010.

[6] B. Bahrani, S. Kenzelmann, and A. Rufer, "Multivariable-PI-based $d q$ current control of voltage source converters with superior axis decoupling capability," IEEE Trans. Ind. Electron., vol. 58, no. 7, pp. 3016-3026, July 2011.

[7] W. Peters and J. Böcker, "Discrete-time design of adaptive current controller for interior permanent magnet synchronous motors (IPMSM) with high magnetic saturation," in Proc. IEEE IECON'13, Vienna, Austria, Nov. 2013, pp. 6608-6613.

[8] M. Hinkkanen, H. A. A. Awan, Z. Qu, T. Tuovinen, and F. Briz, "Current control for synchronous motor drives: direct discrete-time poleplacement design," IEEE Trans. Ind. Appl., vol. 52, no. 2, pp. 15301541, Mar./Apr. 2016.

[9] S. N. Vukosavic, L. S. Peric, and E. Levi, "Digital current controller with error-free feedback acquisition and active resistance," IEEE Trans. Ind. Electron., vol. 65, no. 3, pp. 1980-1990, Sept. 2017.

[10] M. D. Soricellis, D. D. Rú, and S. Bolognani, "A robust current control based on proportional-integral observers for permanent magnet synchronous machines," IEEE Trans. Ind. Appl., vol. 54, no. 2, pp. 14371447, Mar. 2018

[11] A. M. Khambadkone and J. Holtz, "Compensated synchronous PI current controller in overmodulation range and six-step operation of space-vector-modulation based vector-controlled drives," IEEE Trans. Ind. Electron., vol. 49, no. 3, pp. 574-580, Jun. 2002.

[12] Y. Peng, D. Vrancic, and R. Hanus, "Anti-windup, bumpless, and conditioned transfer techniques for PID controllers," IEEE Control Syst. Mag., vol. 16, no. 4, pp. 48-57, Aug. 1996.

[13] H. A. A. Awan, Z. Song, S. E. Saarakkala, and M. Hinkkanen, "Optimal torque control of synchronous motor drives: Plug-and-play method," in Proc. IEEE ECCE, Cincinnati, OH, Oct. 2017, pp. 334-341. 